\title{
ALMOST SURE INVARIANCE PRINCIPLES FOR SUMS OF $B$-VALUED RANDOM VARIABLES WITH APPLICATIONS TO RANDOM FOURIER SERIES AND THE EMPIRICAL CHARACTERISTIC PROCESS
}

BY

\author{
MICHAEL B. MARCUS AND WALTER PHILIPP ${ }^{1,2}$
}

\begin{abstract}
We establish an almost sure approximation of the partial sums of independent, identically distributed random variables with values in a separable Banach space $B$ by a suitable $B$-valued Brownian motion under the hypothesis that the partial sums can be $L^{1}$-closely approximated by finite-dimensional random variables. We show that this hypothesis is satisfied if the given random variables are random Fourier series or related stochastic processes. As an application we obtain an almost sure approximation of the empirical characteristic process by a suitable $\mathbf{C}(K)$-valued Brownian motion whenever the empirical characteristic process satisfies the central limit theorem.
\end{abstract}

1. Introduction. Let $\left\{x_{j}, j \geqslant 1\right\}$ be a sequence of independent identically distributed random variables with values in a real separable Banach space $(B,\|\cdot\|)$ with mean zero and finite second moment. Let $S_{n}$ denote the $n$th partial sum of the sequence. We say that $\left\{x_{j}, j \geqslant 1\right\}$ satisfies the central limit theorem (or that $x=x_{1}$ satisfies the central limit theorem) if $n^{-1 / 2} S_{n}$ converges weakly to a Gaussian measure on $B$.

In [17] it is shown that if $\left\{x_{j}, j \geqslant 1\right\}$ satisfies the central limit theorem then an almost sure invariance principle holds. That is, without changing the probability law of $\left\{x_{j}, j \geqslant 1\right\}$ we can redefine the sequence $\left\{x_{j}, j \geqslant 1\right\}$ on a new probability space on which there exists a $B$-valued Brownian motion $\{X(t), t \geqslant 0\}$ with covariance

$$
E f(X(1)) g(X(1))=E\left(f\left(x_{1}\right) g\left(x_{1}\right)\right), \quad f, g \in B^{*},
$$

such that

$$
\left\|\sum_{j<t} x_{j}-X(t)\right\|=o\left((t \log \log t)^{1 / 2}\right) \quad \text { a.s. }
$$

Received by the editors, September 25, 1980.

1980 Mathematics Subject Classification. Primary 60F15; Secondary 60B99.

Key words and phrases. Invariance principles, Banach space valued random variables, Brownian motion, random Fourier series, empirical characteristic process, central limit theorem.

${ }^{1}$ Both authors were partially supported by NSF grants.

${ }^{2}$ Portions of this paper were presented at the Third International Conference on Probability in Banach Space, Medford, Massachusetts, August 1980. 
Also in [18], under the same hypothesis, an $L^{2}$-invariance principle is obtained, replacing (1.2) by

$$
n^{-1 / 2} \max _{k<n}\left\|\sum_{j<k} x_{j}-X(k)\right\| \rightarrow 0 \text { in } L^{2} .
$$

Let $\left\{P_{s}, 0<s \leqslant 1\right\}$ be a set of uniformly bounded linear operators $P_{s}: B \rightarrow B$ with finite-dimensional range such that

$$
\sup _{n>1} n^{-1 / 2} E\left\|S_{n}-P_{s} S_{n}\right\| \leqslant u(s)
$$

where $\lim _{s \rightarrow 0} u(s)=0$. It is easy to see and it follows also from a well-known theorem of Hoffmann-Jorgensen [7] and Pisier [19] that (1.4) implies the central limit theorem for $\left\{x_{j}, j \geqslant 1\right\}$. Therefore, by the above remarks, (1.4) implies an almost sure and an $L^{2}$-invariance principle, (1.2) and (1.3), as well.

One of our main objectives in this paper is to obtain an estimate of the size of the "little $o$ " term in (1.2) in terms of the function $u$ in (1.4).

THEOREM 1.1. Let $\left\{x_{j}, j \geqslant 1\right\}$ be a sequence of independent, identically distributed $B$-valued random variables, centered at expectations and with finite moments of order $2+\delta$, where $\delta>0$. Let $\left\{P_{s}, 0<s \leqslant 1\right\}$ be a set of uniformly bounded linear operators $P_{s}: B \rightarrow B$ satisfying (1.4). We assume (without serious loss of generality) that $u(s)$ as given in (1.4) is continuous and strictly decreasing as $s \downarrow 0$. Write

$$
u_{1}(s)=u^{\varepsilon}(s) \text { where } \varepsilon=\delta /(3+3 \delta) \text {. }
$$

Then the following three results are obtained:

(i) Let $\operatorname{dim} P_{s} B \leqslant \exp (1 / s)$ and $u_{1}(s)$ satisfy

$$
\frac{u_{1}(s)}{s} \leqslant \frac{u_{1}(t)}{t}, \quad 0<t \leqslant s \leqslant 1,
$$

and $u_{1}(1)=1$. Then without changing its probability law we can redefine the sequence $\left\{x_{j}, j \geqslant 1\right\}$ on a new probability space on which there exists a Brownian motion $\{X(t), t \geqslant 0\}$ with the same covariance structure (1.1) as $x_{1}$ such that with probability 1

$$
\left\|\sum_{j<t} x_{j}-X(t)\right\| \ll g(t, \delta)(t \log \log t)^{1 / 2}
$$

where

$$
g(t, \delta)=(u(\alpha / \log t))^{\varepsilon / 2} \text { and } \alpha=13 / \min (\delta, 1) .
$$

(ii) Let $\operatorname{dim} P_{s} B \leqslant \exp (1 / s)$ and $u(s)=s^{\beta}, 0<\beta<\infty$. Then the conclusion of (i) holds with (1.8) replaced by

$$
g(t, \delta)=(\log t)^{-\beta \varepsilon / 2} .
$$

(iii) Let $\operatorname{dim} P_{s} B \leqslant 1 / s$ and $u(s) \leqslant s^{\beta}, 0<\beta \leqslant 1$. Then the conclusion of (i) holds with (1.8) replaced by

$$
g(t, \delta)=t^{-\lambda}
$$

for some $\lambda>0$, depending on $\delta$ and $\beta$ only. (Note that $f(t) \ll g(t)$ means the same as $f(t)=O(g(t))$ as $t \rightarrow \infty$.) 
A result related to Theorem 1.1 is Theorem 2 of [13].

A few remarks may be useful in explaining Theorem 1.1. In (i) we show that whenever we can find a $u(s)$ satisfying (1.4) we can make some statement about the "little $o$ " term in (1.2) because (1.6) is satisfied by concave functions. Given any $u(s)$ such that $\lim _{s \rightarrow 0} u(s)=0$ we can find a concave function $w_{1}(s)$ satisfying $u_{1}(s) \leqslant w_{1}(s), 0<s \leqslant 1$ and $\lim _{s \rightarrow 0} w_{1}(s)=0$. Therefore, we can use $w_{1}$ in (i) in place of $u_{1}$ and obtain $\ll\left(w_{1}(\alpha / \log t)\right)^{1 / 2}$ as in (1.8) as an improvement over (1.2). Also note that $g(t, \delta) \geqslant(\alpha / \log t)^{\varepsilon / 2}$ in (1.8) because of (1.6). To get smaller bounds we can use (ii) or (iii) when they apply. It would be desirable to get a single expression incorporating all these results and which would be valid for all functions $u$, but we have not been able to do this. Finally, note that the size of $u(s)$ depends on $\operatorname{dim} P_{s}$. Thus $u(s)$ can be much smaller in (iii) than in (ii). This will be clearer in the examples which follow.

Our main application of Theorem 1.1 will be to the Banach space $\mathbf{C}(K, \tau)$ of continuous complex-valued functions on the metric or pseudometric space $(K, \tau)$. Let $N_{\tau}(K, s)$ denote the minimal number of open balls of radius $s$ in the $\tau$-metric or pseudometric with centers in $K$ that cover $K$. Then one can find bounded linear operators $P_{s}$ of dimension $N_{\tau}(K, s)$ (see Lemma 4.1) such that

$$
E\left\|S_{n}-P_{s} S_{n}\right\| \leqslant E\left[\sup _{\tau\left(v, v^{\prime}\right)<s ; v, v^{\prime} \in K}\left|S_{n}(v)-S_{n}\left(v^{\prime}\right)\right|\right] .
$$

Therefore, the term on the right side of (1.11) can be used to obtain $u(s)$ in (1.4). In general, it is not easy to obtain bounds for such expressions. However following, or in some cases slightly modifying, the proofs of some recent results on the central limit theorem in $\mathbf{C}(K, \tau)([8],[14],[15],[16],[6])$, we can obtain such bounds for certain types of stochastic processes. In Theorem 4.2 this is done for four different classes of processes.

To illustrate our results we will present here the one dealing with the empirical characteristic process. It was S. Csörgö's [3] work on almost sure approximation theorems for certain examples of the empirical characteristic process that provided the motivation for this paper. In the following introduction to the empirical characteristic process we follow [3]. Let $X$ be a random variable with values in $\mathbf{R}^{N}$, with distribution function $F(z), z \in \mathbf{R}^{N}$ and characteristic function

$$
c(v)=\int_{\mathbf{R}^{N}} e^{i\langle z, v\rangle} d F(z)=E e^{i\langle X, v\rangle} .
$$

Consider also for $v \in \mathbf{R}^{N}$

$$
\sigma^{2}(v)=2(1-\operatorname{Re} c(v))=4 \int_{\mathbf{R}^{N}} \sin ^{2} \frac{1}{2}\langle z, v\rangle d F(z)
$$

Let $\left\{X_{k}, k \geqslant 1\right\}$ be a sequence of independent copies of $X$. The empirical distribution function of $X$, based on a sample of size $n$, is

$$
F_{n}(z)=n^{-1} \sum_{j<n} 1\left\{X_{j} \leqslant z\right\}
$$


The Fourier transform of $F_{n}$ equals

$$
c_{n}(v)=n^{-1} \sum_{j<n} e^{1\left\langle X_{j}, v\right\rangle}
$$

and is called the empirical characteristic function of $X$. The empirical characteristic process is defined as

$$
C(v, t)=\sum_{j<t}\left(e^{i\left\langle X_{j}, v\right\rangle}-c(v)\right), \quad v \in\left[-\frac{1}{2}, \frac{1}{2}\right]^{N}, t \geqslant 0 .
$$

It is necessary to restrict attention to compact subsets of $\mathbf{R}^{N}$ (see [3] for details).

In view of (1.15) the question of weak convergence of $n^{-1 / 2} C(\cdot, n)$ is the same as that of the central limit theorem for $e^{i\langle X, v\rangle}-c(v)$. If the sequence $\left\{n^{-1 / 2} C(\cdot, n)\right.$, $n \geqslant 1\}$ converges weakly on $\mathbf{C}\left(\left[-\frac{1}{2}, \frac{1}{2}\right]^{N}\right)$ the limit must be a Gaussian process $G$, with continuous sample paths and with covariance

$$
E\left\{\left(e^{i\langle X, v\rangle}-c(v)\right)\left(e^{-i\left\langle X, v^{\prime}\right\rangle}-c\left(-v^{\prime}\right)\right)\right\}=c\left(v-v^{\prime}\right)-c(v) c\left(-v^{\prime}\right) .
$$

The spectral representation of this process is

$$
G(v)=\int_{\mathbf{R}^{N}} e^{i\langle z, v\rangle} d(b(F(z))-b(1) c(v)),
$$

where $b$ is standard Brownian motion. Therefore in order for $n^{-1 / 2} C(\cdot, n)$ to have a weak limit in $\mathbf{C}\left(\left[-\frac{1}{2}, \frac{1}{2}\right]^{N}\right)$ the stationary Gaussian process

$$
\int_{\mathbf{R}^{N}} e^{i\langle z, v\rangle} d b(F(z)), \quad v \in\left[-\frac{1}{2}, \frac{1}{2}\right]^{N},
$$

must have a version with continuous paths. By the Dudley-Fernique theorem this happens if and only if

$$
\int_{0}^{\infty}\left(\log N_{\sigma}\left(\left[-\frac{1}{2}, \frac{1}{2}\right]^{N}, \varepsilon\right)\right)^{1 / 2} d \varepsilon<\infty
$$

for $\sigma$ given in (1.13) (see [15, relations (3), (4) and (5)]). It was shown in [15], in the case $N=1$, that whenever $(1.16)$ holds $n^{-1 / 2} C(\cdot, n)$ does converge weakly to a Gaussian limit. The result for $N>1$ has the same proof as the result for $N=1$. It is given in Theorem 4.2 and Remark 4.3 along with central limit theorems for many related stochastic processes.

Consequently, if (1.16) holds then in view of (1.2) and (1.3)

$$
\sup _{v \in\left[-\frac{1}{2}, \frac{1}{2}\right]^{N}}|C(v, t)-H(v, t)|=o\left((t \log \log t)^{1 / 2}\right) \text { a.s. }
$$

and $o\left(t^{1 / 2}\right)$ in $L^{2}$, where $\left\{H(v, t), v \in\left[-\frac{1}{2}, \frac{1}{2}\right]^{N}, t \geqslant 0\right\}$ is a Gaussian process with mean zero and convariance function

$$
E\left(H(v, t) \overline{H\left(v^{\prime}, t\right)}\right)=\min \left(t, t^{\prime}\right)\left(c\left(v-v^{\prime}\right)-c(v) c\left(-v^{\prime}\right)\right) .
$$

In Theorem 1.2 which is an application of Theorem 1.1 we refine (1.17). Let

$$
\begin{gathered}
R(x)=P(|X| \leqslant x), \\
\hat{\sigma}(s)=2\left(s^{2} \int_{0}^{1 / s} x(1-R(x)) d x\right)^{1 / 2},
\end{gathered}
$$


and

$$
\psi(s)=\int_{0}^{\hat{\sigma}(s)}\left(\log N_{\sigma}\left(\left[-\frac{1}{2}, \frac{1}{2}\right]^{N}, \varepsilon\right)\right)^{1 / 2} d \varepsilon+\hat{\sigma}(s) .
$$

THEOREM 1.2. Let $\left\{X_{j}, j \geqslant 1\right\}$ be a sequence of independent identically distributed random variables with values in $\mathbf{R}^{N}$ and distribution function $F$. Employing the notation given above, we can redefine the sequence $\left\{X_{j}, j \geqslant 1\right\}$ without changing its probability law, on a new probability space on which there exists a mean zero Gaussian process $\left\{H(v, t), v \in\left[-\frac{1}{2}, \frac{1}{2}\right]^{N}, t \geqslant 0\right\}$ with covariance function (1.18) such that with probability 1

$$
\sup _{v \in\left[-\frac{1}{2}, \frac{1}{2}\right]^{N}}|C(v, t)-H(v, t)| \ll w^{1 / 2}\left(B_{N} t^{-13 N}\right)(t \log \log t)^{1 / 2} .
$$

Here $w(s)$ is a concave majorant of $\psi^{1 / 4}\left(B_{N} \exp \left(-(s N)^{-1}\right)\right), B_{N}$ is a constant depending on $N$ only and $\psi$ is given in (1.21). If

$$
1-R(x) \ll 1 / \log x(\log \log x)^{2 g}, \quad g>1,
$$

then the error term in (1.22) is

$$
\ll(\log \log t)^{-(g-1) / 8}(t \log \log t)^{1 / 2} .
$$

If

$$
1-R(x) \ll(\log x)^{-g}, \quad g>1,
$$

then the error term in (1.22) is

$$
\ll(\log t)^{-(g-1) / 8}(t \log \log t)^{1 / 2} .
$$

If

$$
1-R(x) \ll x^{-g}, \quad g>0,
$$

then the error term in (1.22) can be replaced by a term which is

$$
\ll t^{1 / 2-\lambda}
$$

for some $\lambda>0$, depending only on $g$ and $N$.

We add a few remarks concerning (1.22). Since (1.16) is necessary and sufficient for the central limit theorem to hold for the sequence $\left\{e^{i\left\langle X_{j}, v\right\rangle}-c(v) ; j \geqslant 1\right.$, $\left.v \in\left[-\frac{1}{2}, \frac{1}{2}\right]^{N}\right\}$ we see from (1.21) and (1.22) that whenever the central limit theorem does hold for this sequence we obtain an estimate for the "little $o$ " term in (1.17). In the rest of Theorem 1.2 using smooth upper bounds for $1-R(x)$ we can obtain cleaner results. To help interpret all this let us note that if $1-R(x)$ is convex for $x$ sufficiently large then (1.16) holds if and only if

$$
\int_{2}^{\infty} \frac{(1-R(x))^{1 / 2}}{x(\log x)^{1 / 2}} d x<\infty
$$

(see Theorem 3.2, Chapter 4, of [10]). Thus the bounds in (1.23) and (1.25) are about as large as they possibly can be. Actually, bounds can be found for all slowly varying $1-R(x)$ satisfying (1.29). The case considered in (1.27) and (1.28) with a sharper estimate of the error term is due to S. Csörgö [3] for $N=1$; for $N>1$ it 
was obtained independently by him also with a sharper estimate of the error term. Some other applications of Theorem 1.1 as well as the proof of Theorem 1.2 will be given in $\$ 4$. The proof of Theorem 1.1 is given in $\$ 3 . \$ 2$ contains the preliminaries. Some further applications of these results are mentioned in $\$ 5$.

2. Preliminaries. For the proof of Theorem 1.1 we need several theorems which we will quote for the reader's convenience. Recall that the Prohorov distance $\pi(\mu, \nu)$ of two probability measures $\mu$ and $\nu$ on a separable metric space $(S, \sigma)$ is defined as

$$
\pi(\mu, \nu)=\inf \left\{\varepsilon>0: \mu(A) \leqslant \nu\left(A^{\varepsilon}\right)+\varepsilon \text { for all closed subsets } A \text { of } S\right\} .
$$

Here $A^{\varepsilon}=\{x \in S: \sigma(x, A)<\varepsilon\}$. The following result is a special case of Dehling's [4] recent generalization of a theorem of Yurinskii [20].

THEOREM 2.1. Let $\left\{\xi_{j}, j \geqslant 1\right\}$ be a sequence of independent, identically distributed random variables in a d-dimensional Banach space $B$, centered at expectations and with finite $(2+\gamma)$ moments where $0<\gamma \leqslant 1$. Let $S_{n}=\Sigma_{j \leqslant n} \xi_{j}$, let $\mu_{n}$ denote the distribution of $n^{-1 / 2} S_{n}$ and let $\mu$ be the mean zero Gaussian measure with covariance function $E\left\{f\left(\xi_{1}\right) g\left(\xi_{1}\right)\right\}, f, g \in B^{*}$. Then

$$
\pi\left(\mu_{n}, \mu\right) \leqslant C d^{4 / 3} n^{-\gamma / 9}
$$

where $C$ only depends on $E\left|\xi_{j}\right|^{2+\gamma}$.

To avoid misunderstandings a few remarks might be in order. Yurinskii's [20] Theorem 1 deals with independent random variables assuming values in a finitedimensional inner product space (i.e. a space where the distance is given by an inner product) and having finite third moments. Dehling's [4] Theorem 1 partially generalizes Yurinskii's theorem to weakly stationary absolutely regular sequences of finite-dimensional random variables having uniformly bounded moments of order $2+\gamma$. Theorem 2.1 follows at once from Dehling's [4] Proposition 5.1 and Lemma 5.1. Except for the exponents $4 / 3$ and $-\gamma / 9$ it is also contained in his Theorem la.

We also need the following result which is given in a more general setting as Theorem 3 in [17].

THEOREM 2.2. Let $\left\{B_{k}, m_{k}, k \geqslant 1\right\}$ be a sequence of complete separable metric spaces. Let $\left\{Z_{k}, k \geqslant 1\right\}$ be a sequence of independent random variables with values in $B_{k}$. Denote the distribution of $Z_{k}$ by $F_{k}$ and let $\left\{G_{k}, k \geqslant 1\right\}$ be a sequence of distributions on $B_{k}$ such that the Prohorov distance $\pi\left(F_{k}, G_{k}\right) \leqslant \rho_{k}, \rho_{k}>0$. Then without changing its distribution we can redefine the sequence $\left\{Z_{k}, k \geqslant 1\right\}$ on a richer probability space on which there exists a sequence $\left\{Y_{k}, k \geqslant 1\right\}$ of independent random variables $Y_{k}$ with distribution $G_{k}$ such that for all $k \geqslant 1$

$$
P\left\{m_{k}\left(Z_{k}, Y_{k}\right) \geqslant 2 \rho_{k}\right\} \leqslant 2 \rho_{k} \text {. }
$$

The following theorem is due to Kuelbs [12, Lemma 2.1]. 
THEOREM 2.3. Let $\left\{\xi_{j}, j \geqslant 1\right\}$ be a sequence of independent $B$-valued random variables, centered at expectations and satisfying $\left\|\xi_{j}\right\| \leqslant c b_{n}(1 \leqslant j \leqslant n)$ where $c$ and $b_{n}$ are constants. Let $T_{n}=\Sigma_{j \leqslant n} \xi_{j}$. Then for any $\rho$ with $\rho c \leqslant 1$,

$$
P\left\{\left\|T_{n}\right\| \geqslant 2 \rho b_{n}\right\} \leqslant \exp \left\{-\rho^{2}+\frac{1}{2} \rho^{2}\left(1+\frac{1}{2} \rho c\right) b_{n}^{-2} \sum_{j<n} E\left\|\xi_{j}\right\|^{2}+\frac{1}{2} \rho b_{n}^{-1} E\left\|T_{n}\right\|\right\} .
$$

\section{Proof of Theorem 1.1.}

3.1. Case (i). By (1.6) and the fact that $u_{1}(1)=1$ we have

$$
u_{1}(s) \geqslant s \text {. }
$$

Consider the function $\left(s u_{1}(s)\right)^{-1}$. This function is continuous and strictly decreasing for $0<s<1$. We define the strictly decreasing sequence $\left\{s_{k}\right\}, \lim _{s \rightarrow \infty} s_{k}=0$ as the unique solution of the equation

$$
k=\alpha\left(s_{k} u_{1}\left(s_{k}\right)\right)^{-1} .
$$

Note that

$$
u_{1}\left(s_{k}\right) \geqslant(\alpha / k)^{1 / 2}
$$

since by $(3.1) u_{1}\left(s_{k}\right) \geqslant s_{k}=\alpha /\left(k u_{1}\left(s_{k}\right)\right)$. We also define

$$
t_{k}=\exp \left(\alpha / s_{k}\right)
$$

and

$$
n_{k, \gamma}=t_{k}^{\gamma}-t_{k-1}^{\gamma}, \quad 0<\gamma \leqslant 1 .
$$

For simplicity we denote $n_{k, 1}=n_{k}$.

There are a number of estimates needed in the proof of Theorem 1.1 which we give in the next lemma.

\section{LEMMA 3.1. We have}

$$
\begin{gathered}
u_{1}\left(s_{k}\right) /(2 \alpha) \leqslant s_{k}^{-1}-s_{k-1}^{-1} \leqslant u_{1}\left(s_{k}\right) / \alpha, \\
t_{k}^{\gamma} u_{1}\left(s_{k}\right) \ll n_{k, \gamma} \ll t_{k}^{\gamma} u_{1}\left(s_{k}\right), \quad 0<\gamma \leqslant 1, \\
\sum_{k<m} n_{k}^{1 / 2} u_{1}^{3 / 2}\left(s_{k}\right) \ll t_{m}^{1 / 2} u_{1}\left(s_{m}\right), \\
n_{k}^{-\tau} t_{k}^{\sigma} \ll t_{k}^{(\sigma-\tau) / 2} \ll \exp \left(-k^{1 / 4}\right), \quad 0 \leqslant \sigma<\tau, \\
\sum_{k<m} t_{k}^{1 / 2-\lambda} \ll t_{m}^{1 / 2-\lambda / 2}, \quad 0<\lambda<\frac{1}{2} .
\end{gathered}
$$

Proof. We begin with (3.6). By (3.2)

$$
\begin{aligned}
\frac{1}{\alpha} & =\frac{1}{s_{k} u_{1}\left(s_{k}\right)}-\frac{1}{s_{k-1} u_{1}\left(s_{k-1}\right)} \\
& =\frac{\left(s_{k-1}-s_{k}\right) u_{1}\left(s_{k-1}\right)-s_{k}\left(u_{1}\left(s_{k}\right)-u_{1}\left(s_{k-1}\right)\right)}{s_{k} s_{k-1} u_{1}\left(s_{k-1}\right) u_{1}\left(s_{k}\right)} \\
& \leqslant\left(s_{k}^{-1}-s_{k-1}^{-1}\right)\left(\left(u_{1}\left(s_{k}\right)\right)^{-1}+\left(u_{1}\left(s_{k-1}\right)\right)^{-1}\right) \leqslant 2\left(s_{k}^{-1}-s_{k-1}^{-1}\right) / u_{1}\left(s_{k}\right)
\end{aligned}
$$


which gives the left side of (3.6). Note that we have used

$$
\left(u_{1}\left(s_{k-1}\right)-u_{1}\left(s_{k}\right)\right) / u_{1}\left(s_{k}\right) \leqslant\left(s_{k-1}-s_{k}\right) / s_{k}
$$

which is an immediate consequence of (1.6). For the right side of (3.6) we simply observe that by the first two lines of (3.11)

$$
\frac{1}{\alpha} \geqslant \frac{s_{k-1}-s_{k}}{s_{k} s_{k-1} u_{1}\left(s_{k}\right) u_{1}\left(s_{k-1}\right)}=\left(s_{k}^{-1}-s_{k-1}^{-1}\right) / u_{1}\left(s_{k}\right) .
$$

By (3.6) we see that for $k$ sufficiently large $s_{k}^{-1}-s_{k-1}^{-1}$ is small; thus by (3.4) and (3.5)

$$
n_{k, \gamma}=t_{k}^{\gamma}-t_{k-1}^{\gamma} \sim t_{k}^{\gamma} \alpha\left(s_{k}^{-1}-s_{k-1}^{-1}\right)
$$

and (3.7) follows from (3.6).

We proceed to obtain (3.8). By (3.5) and (3.7) we have

$$
\sum_{k<m} t_{k}^{\gamma} u_{1}\left(s_{k}\right) \ll t_{m}^{\gamma}=\sum_{k<m} n_{k}^{\gamma} \ll \sum_{k<m} t_{k}^{\gamma} u_{1}\left(s_{k}\right)
$$

Since by (3.2)

$$
(k+1) / \alpha=\left(s_{k+1} u_{1}\left(s_{k+1}\right)\right)^{-1} \geqslant\left(s_{k} u_{1}\left(s_{k}\right)\right)^{-1} \cdot s_{k} / s_{k+1} \geqslant(k / \alpha)\left(s_{k} / s_{k+1}\right),
$$

we obtain

$$
s_{k} / s_{k+1} \leqslant 1+1 / k
$$

Hence by (3.12) and (3.3)

$$
u_{1}\left(s_{k}\right)-u_{1}\left(s_{k+1}\right) \leqslant u_{1}\left(s_{k}\right) \frac{s_{k}-s_{k+1}}{s_{k+1}} \leqslant u_{1}\left(s_{k}\right) / k \leqslant u_{1}^{3}\left(s_{k}\right) / \alpha .
$$

By summation by parts and (3.13) we thus obtain

$$
\begin{aligned}
\sum_{k<m} t_{k}^{1 / 2} u_{1}^{2}\left(s_{k}\right)= & \left(\sum_{k<m} t_{k}^{1 / 2} u_{1}\left(s_{k}\right)\right) u_{1}\left(s_{m}\right) \\
& +\sum_{k<m}\left(\sum_{j<k} t_{j}^{1 / 2} u_{1}\left(s_{j}\right)\right)\left(u_{1}\left(s_{k}\right)-u_{1}\left(s_{k+1}\right)\right) \\
\ll & t_{m}^{1 / 2} u_{1}\left(s_{m}\right)+\sum_{k<m} t_{k}^{1 / 2} u_{1}^{3}\left(s_{k}\right) \ll t_{m}^{1 / 2} u_{1}\left(s_{m}\right)
\end{aligned}
$$

since $u_{1}\left(s_{k}\right) \rightarrow 0$. Inequality (3.8) follows now from (3.7) in the case $\gamma=1$.

Next we note that by (3.2) and (3.3)

$$
(\alpha k)^{1 / 2} \leqslant \alpha / s_{k} \leqslant k
$$

so that

$$
e^{(\alpha k)^{1 / 2}} \leqslant t_{k} \leqslant e^{k}
$$

Hence by (3.7) and (3.3) we get (3.9). For (3.10) we simply note that

$$
\sum_{k<m} t_{m}^{1 / 2-\lambda} \leqslant m t_{m}^{1 / 2-\lambda} \ll t_{m}^{1 / 2-\lambda / 2}, \quad 0<\lambda<\frac{1}{2} .
$$

This completes the proof of Lemma 3.1. 
LEMMA 3.2. As $k \rightarrow \infty$,

$$
\left\|\sum_{t_{k-1}<j<t_{k}}\left(x_{j}-P_{s_{k}} x_{j}\right)\right\| \ll u\left(s_{k}\right)^{3 \varepsilon / 2}\left(n_{k} \log \log t_{k}\right)^{1 / 2} \quad \text { a.s. }
$$

Proof. We write

$$
E\left\|x_{j}-P_{s} x_{j}\right\|^{2}=E\left\{\left\|x_{j}-P_{s} x_{j}\right\|^{\delta /(1+\delta)}\left\|x_{j}-P_{s} x_{j}\right\|^{(2+\delta) /(1+\delta)}\right\} .
$$

Then by Hölder's inequality with $p=(1+\delta) / \delta$ and $q=1+\delta$ we get

$$
E\left\|x_{j}-P_{s} x_{j}\right\|^{2} \leqslant\left(E\left\|x_{j}-P_{s} x_{j}\right\|\right)^{\delta /(1+\delta)}\left(E\left\|x_{j}-P_{s} x_{j}\right\|^{2+\delta}\right)^{1 /(1+\delta)} .
$$

Let $M=1+\sup _{0<s \leqslant 1}\left\|P_{s}\right\|$. We always can normalize so that

$$
M \cdot E\left\|x_{1}\right\|^{2+\delta} \leqslant 1
$$

Thus by (1.4) and (1.5)

$$
E\left\|x_{j}-P_{s} x_{j}\right\|^{2} \leqslant u(s)^{3 \varepsilon}
$$

Put

$$
z_{j}=z_{j}(s)=x_{j}-P_{s} x_{j}
$$

Before we apply Theorem 2.3 we have to truncate the $z_{j}$ 's and to center the truncated random variables. We put

$$
z_{j}^{\prime}=z_{j} 1\left\{\left\|x_{j}\right\| \leqslant j^{1 /(2+\delta)}\right\}
$$

Then by (3.17)

$$
E\left\|z_{j}^{\prime}\right\|^{2} \leqslant E\left\|z_{j}\right\|^{2} \leqslant u(s)^{3 \varepsilon} .
$$

Since $E z_{j}=0$ we have by (3.16)

$$
\begin{aligned}
\left\|E z_{j}^{\prime}\right\| & =\left\|E\left\{z_{j} 1\left\{\left\|x_{j}\right\|>j^{1 /(2+\delta)}\right\}\right\}\right\| \\
& \leqslant M E\left\|x_{j}\right\|^{2+\delta_{j}-(1+\delta) /(2+\delta)} .
\end{aligned}
$$

Thus by (3.5), (3.7) and (3.9)

$$
\begin{aligned}
\sum_{t_{k-1}<j<t_{k}}\left\|E z_{j}^{\prime}\right\| & \ll t_{k}^{1 /(2+\delta)}-t_{k-1}^{1 /(2+\delta)} \ll t_{k}^{1 /(2+\delta)} u\left(s_{k}\right)^{3 \varepsilon / 2} \\
& \ll n_{k}^{1 / 2-\lambda} u\left(s_{k}\right)^{3 \varepsilon / 2}
\end{aligned}
$$

for some $\lambda>0$. Similarly, since $z_{j}-z_{j}^{\prime}=z_{j} 1\left\{\left\|x_{j}\right\|>j^{1 /(2+\delta)}\right\}$,

$$
E\left\|\sum_{t_{k-1}<j<t_{k}} z_{j}-z_{j}^{\prime}\right\| \leqslant \sum_{t_{k-1}<j<t_{k}} E\left\|z_{j}-z_{j}^{\prime}\right\| \ll n_{k}^{1 / 2-\lambda} u\left(s_{k}\right)^{3 e / 2} .
$$

Thus by (1.4)

$$
E\left\|\sum_{t_{k-1}<j<t_{k}} z_{j}^{\prime}\right\| \ll n_{k}^{1 / 2} u\left(s_{k}\right)^{3 \varepsilon / 2}
$$


We choose $b_{k}=4 n_{k}^{1 / 2} u\left(s_{k}\right)^{3 \varepsilon / 2}, \quad c=c_{k}=(M / 2) t_{k}^{-\delta /(8+4 \delta} u\left(s_{k}\right)^{-3 \varepsilon / 2}$ and $\rho=$ $4\left(\log \log t_{k}\right)^{1 / 2}$ in Theorem 2.3 and obtain for sufficiently large $k$

$$
\begin{aligned}
& P\left\{\left\|\sum_{t_{k-1}<j<t_{k}} z_{j}^{\prime}\left(s_{k}\right)\right\| \geqslant 9 \rho n_{k}^{1 / 2} u\left(s_{k}\right)^{3 \varepsilon / 2}\right\} \\
& \leqslant P\left\{\left\|\sum_{t_{k-1}<j<t_{k}} z_{j}^{\prime}\left(s_{k}\right)-E z_{j}^{\prime}\left(s_{k}\right)\right\| \geqslant 8 \rho n_{k}^{1 / 2} u\left(s_{k}\right)^{3 \varepsilon / 2}\right\} \\
& \leqslant \exp \left(-\rho^{2}+\frac{1}{2} \rho^{2}(1+o(1)) / 16+\rho \cdot o(1)\right) \ll \exp \left(-\frac{1}{2} \rho^{2}\right) \ll k^{-2}
\end{aligned}
$$

by (3.3), (3.7), (3.9) and (3.15). Hence by the Borel Cantelli lemma

$$
\left\|\sum_{t_{k-1}<j<t_{j}} z_{j}^{\prime}\right\| \ll\left(n_{k} \log \log t_{k}\right)^{1 / 2} u\left(s_{k}\right)^{3 \varepsilon / 2} \text { a.s. }
$$

It remains to show that

$$
\sum_{k>1} P\left\{z_{j}^{\prime} \neq z_{j} \text { for some } j \text { with } t_{k-1}<j \leqslant t\right\}<\infty .
$$

Then an application of the Borel Cantelli lemma and the last inequality will give

$$
\left\|\sum_{t_{k-1}<j<t_{k}} z_{j}\right\| \ll\left(n_{k} \log \log t_{k}\right)^{1 / 2} u\left(s_{k}\right)^{3 \varepsilon / 2} \quad \text { a.s., }
$$

the conclusion of the lemma. We have

$$
\begin{aligned}
\sum_{t_{k-1}<j<t_{k}} P\left\{z_{j}^{\prime} \neq z_{j}\right\} & \leqslant \sum_{t_{k-1}<j<t_{k}} P\left\{\left\|x_{j}\right\|>j^{1 /(2+\delta)}\right\} \\
& =\sum_{t_{k-1}<j<t_{k}} P\left\{\left\|x_{1}\right\|^{2+\delta}>j\right\} .
\end{aligned}
$$

We sum these last terms over $k \geqslant 1$ and obtain a convergent series since $E\left\|x_{1}\right\|^{2+\delta}$ $<\infty$. This concludes the proof of the lemma.

By the remark following (1.4) the sequence $\left\{x_{j}, j \geqslant 1\right\}$ satisfies the central limit theorem. Hence there exists a Gaussian measure $\mu$ with the same covariance as $x_{1}$. Let $\{X(t), t \geqslant 0\}$ be a $B$-valued Brownian motion with $L(X(1))=\mu$.

LEMMA 3.3. As $k \rightarrow \infty$

$$
\left\|X\left(t_{k}\right)-X\left(t_{k-1}\right)-P_{s_{k}}\left(X\left(t_{k}\right)-X\left(t_{k-1}\right)\right)\right\| \ll u\left(s_{k}\right)^{3 \varepsilon / 2}\left(n_{k} \log \log t_{k}\right)^{1 / 2} \quad \text { a.s. }
$$

Proof. As we noted above $\left\{x_{j}, j \geqslant 1\right\}$ satisfies the central limit theorem. Hence the measures induced on $B$ by $n^{-1 / 2}\left(S_{n}-P_{s} S_{n}\right)$ converge weakly to $X(1)-P_{s} X(1)$. Since $\left\{x \in B:\left\|x-P_{s} x\right\|>\tau\right\}$ is an open set in $B$ we have by (1.4) and a standard theorem on the weak convergence of probability measures that

$$
\begin{aligned}
& P\left\{\left\|X(1)-P_{s} X(1)\right\|>3 u(s)\right\} \\
& \quad \leqslant \liminf _{n \rightarrow \infty} P\left\{n^{-1 / 2}\left\|S_{n}-P_{s} S_{n}\right\|>3 u(s)\right\}<\frac{1}{3} .
\end{aligned}
$$

Since $X(1)-P_{s} X(1)$ is a bounded Banach space valued Gaussian random variable we obtain from the Fernique, Landau-Shepp lemma

$$
P\left\{\left\|X(1)-P_{s} X(1)\right\|>\rho u(s)\right\} \leqslant \exp \left(-\delta^{2} / \alpha^{2}\right) \quad \text { for some } \alpha<\infty \text { and all } \rho \geqslant 1 \text {. }
$$


By the nature of Brownian motion this inequality is equivalent to

$$
P\left\{\left\|X(n)-P_{s} X(n)\right\|>\rho n^{1 / 2} u(s)\right\} \leqslant \exp \left(-\rho^{2} / \alpha^{2}\right) .
$$

We put $\rho=4 \alpha\left(\log \log t_{k}\right)^{1 / 2}, s=s_{k}$ and $n=n_{k}$ and using the fact that $L\left(X\left(n_{k}\right)\right)=$ $L\left(X\left(t_{k}\right)-X\left(t_{k-1}\right)\right)$ and (3.15) we obtain the result via the Borel Cantelli lemma.

LeMma 3.4. As $k \rightarrow \infty$

$$
\sup _{t_{k-1}<t<t_{k}}\left\|\sum_{t_{k-1}<j<t} x_{j}\right\| \ll u\left(s_{k}\right)^{\varepsilon / 2}\left(t_{k} \log \log t_{k}\right)^{1 / 2} \quad \text { a.s. }
$$

Proof. Again we truncate the random variables $x_{j}$ at $j^{1 /(2+\delta)}$. Set

$$
x_{j}^{\prime}=x_{j} 1\left\{\left\|x_{j}\right\|<j^{1 /(2+\delta)}\right\}, \quad x_{j}^{\prime \prime}=x_{j}-x_{j}^{\prime},
$$

and

$$
S_{n}^{\prime}=\sum_{j<n} x_{j}^{\prime}
$$

Since $E\left\|x_{1}\right\|^{2+\delta}<\infty$ we have by stationarity

$$
\sum_{j>1} P\left(x_{j}^{\prime \prime} \neq 0\right)=\sum_{j>1} P\left(\left\|x_{j}\right\|>j^{1 /(2+\delta)}\right)<\infty
$$

and thus by the Borel Cantelli lemma

$$
\sum_{j>1}\left\|x_{j}^{\prime \prime}\right\|<\infty \quad \text { a.s. }
$$

Now by (3.7) there exists a constant $C$ such that

$$
\begin{aligned}
\max _{t_{k-1}<n<t_{k}} P\{ & \left.\left\|S_{n}^{\prime}-S_{t_{k-1}}^{\prime}\right\| \geqslant C u\left(s_{k}\right)^{\varepsilon / 2}\left(t_{k} \log \log t_{k}\right)^{1 / 2}\right\} \\
& \leqslant \max _{t_{k-1}<n<t_{k}} P\left\{\left(n-t_{k-1}\right)^{-1 / 2}\left\|S_{n}^{\prime}-S_{t_{k-1}}^{\prime}\right\|\right. \\
& \left.\geqslant 4\left(n_{k} \log \log t_{k} /\left(n-t_{k-1}\right)\right)^{1 / 2}\right\} \\
& \leqslant \max _{t_{k-1}<n<t_{k}} P\left\{\left(n-t_{k-1}\right)^{-1 / 2}\left\|S_{n}^{\prime}-S_{t_{k-1}}^{\prime}\right\| \geqslant 4\left(\log \log t_{k}\right)^{1 / 2}\right\} .
\end{aligned}
$$

In view of (3.19) $\left\{x_{j}^{\prime}, j \geqslant 1\right\}$ satisfies the central limit theorem since $\left\{x_{j}, j \geqslant 1\right\}$ does. Thus the last term in (3.20) goes to zero as $k \rightarrow \infty$. We refer the reader to Lemma 3.21 of [2], p. 45. This lemma is proved for real-valued random variables; however, the extension to $B$-valued random variables is immediate. Hence we obtain for some constant $D$

$$
\begin{aligned}
P\left\{\max _{t_{k-1}<t<t_{k}}\left\|S_{t}^{\prime}-S_{t_{k-1}}^{\prime}\right\|\right. & \left.\geqslant 32 u\left(s_{k}\right)^{\varepsilon / 2}\left(D t_{k} \log \log t_{k}\right)^{1 / 2}\right\} \\
\ll & \ll\left\{\left\|S_{t_{k}}^{\prime}-S_{t_{k-1}}^{\prime}\right\| \geqslant 16 u\left(s_{k}\right)^{\varepsilon / 2}\left(D t_{k} \log \log t_{k}\right)^{1 / 2}\right\} \\
& \ll P\left\{\left\|S_{t_{k}}^{\prime}-S_{t_{k-1}}^{\prime}\right\| \geqslant 16\left(n_{k} \log \log t_{k}\right)^{1 / 2}\right\}
\end{aligned}
$$

by (3.7). Since $E\left\|P_{1}\left(S_{t_{k}}-S_{t_{k-1}}\right)\right\| \ll n_{k}^{1 / 2}$ we obtain by (1.4) with $s=1$,

$$
E\left\|S_{t_{k}}-S_{t_{k-1}}\right\| \ll n_{k}^{1 / 2} \text {. }
$$


Now

$$
\begin{aligned}
E \| S_{t_{k}}-S_{t_{k-1}}- & \left(S_{t_{k}}^{\prime}-S_{t_{k-1}}^{\prime}\right) \| \\
& \leqslant \sum_{t_{k-1}<j<t_{k}} E\left\|x_{j}^{\prime \prime}\right\|=\sum_{t_{k-1}<j<t_{k}} E\left\{\left\|x_{j}\right\| 1\left(\left\|x_{j}\right\| \geqslant j^{1 /(2+\delta)}\right)\right\} \\
& \leqslant \sum_{t_{k-1}<j<t_{k}} E\left\|x_{1}\right\|^{2+\delta} j^{-(1+\delta) /(2+\delta)} \ll n_{k}^{1 /(2+\delta)} .
\end{aligned}
$$

Hence

$$
E\left\|S_{t_{k}}^{\prime}-S_{t_{k-1}}^{\prime}\right\| \ll n_{k}^{1 / 2} \text {. }
$$

We now can find an upper bound for the last term in (3.21) using Theorem 2.3 with $b_{k}=2 n_{k}^{1 / 2}, c=c_{k}=n_{k}^{-\delta /(8+4 \delta)}$ and $\rho=4\left(\log \log t_{k}\right)^{1 / 2}$. By (3.3), (3.7) and (3.15) it follows that $\rho c \rightarrow 0$. Thus we obtain for the probability in (3.21) the bound $\ll k^{-2}$ using (3.15) once more. The lemma follows now from the Borel Cantelli lemma and (3.19).

LeMma 3.5. As $k \rightarrow \infty$,

$$
\sup _{t_{k-1}<t \leqslant t_{k}}\left\|X(t)-X\left(t_{k}\right)\right\| \ll u\left(s_{k}\right)^{\varepsilon / 2}\left(t_{k} \log \log t_{k}\right)^{1 / 2} \quad \text { a.s. }
$$

Proof. Since $X(t)$ is a Brownian motion

$$
\begin{aligned}
\sup _{t_{k-1}<t<t_{k}}\left\|X(t)-X\left(t_{k}\right)\right\| \leqslant & \sup _{t_{k-1}<j<t_{k}}\left\|X(j)-X\left(\left[t_{k-1}\right]+1\right)\right\| \\
& +2 \sup _{0<t<1}\|X(t)\|,
\end{aligned}
$$

where $j$ is an integer and [ $\cdot]$ denotes integral part. The first term in (3.22) is estimated exactly as in Lemma 3.4 (or by applying Lemma 3.4 to the increments $X(j)-X(j-1))$ and the second term in (3.22) is just a finite random variable.

We have already estimated various quantities involving the limiting Brownian motion. Now we must construct a probability space on which the sequence $\left\{x_{j}\right.$, $j \geqslant 1\}$ and the Brownian motion $\{X(t), t \geqslant 0\}$ are defined in order to give a meaning to (1.7). Consider

$$
Z_{k}=n_{k}^{-1 / 2} \sum_{t_{k-1}<j<t_{k}} P_{s_{k}} x_{j}
$$

and denote by $d_{k}$ the dimension of $P_{s_{k}}$. In (i) $d_{k} \leqslant \exp \left(1 / s_{k}\right)$. Let $F_{k}$ denote the distribution of $Z_{k}$ and let $G_{k}$ denote the distribution of a mean zero Gaussian vector of dimension $d_{k}$ and with the same covariance matrix as $Z_{k}$. From Theorem $2.1,(3.4)$ (1.8) and (3.9)

$$
\rho_{k}=\pi\left(F_{k}, G_{k}\right) \ll n_{k}^{-\delta / 9} \exp \left(4 / 3 s_{k}\right) \ll t_{k}^{-\lambda_{1}} \ll \exp \left(-k^{1 / 4}\right)
$$

for some $\lambda_{1}>0$. Therefore, by Theorem 2.2 there exists without loss of generality, a sequence of independent random vectors $Y_{k}$ with distribution $G_{k}$ such that $P\left\{\left\|Z_{k}-Y_{k}\right\| \geqslant 2 \rho_{k}\right\} \leqslant 2 \rho_{k}$. Hence by (3.5), (3.24) and the Borel Cantelli lemma

$$
\left\|n_{k}^{1 / 2} Z_{k}-\left(t_{k}-t_{k-1}\right)^{1 / 2} Y_{k}\right\| \ll t_{k}^{1 / 2-\lambda_{1}} \text { a.s. }
$$


We now construct the desired Brownian motion. We first observe that for any $B$-valued Brownian motion $\{X(t), t \geqslant 0\}$ with covariance function given by (1.1) the sequences of $d_{k}$-dimensional random vectors

$$
\left\{\left(t_{k}-t_{k-1}\right)^{-1 / 2} P_{s_{k}}\left(X\left(t_{k}\right)-X\left(t_{k-1}\right)\right), k \geqslant 1\right\} \text { and }\left\{Y_{k}, k \geqslant 1\right\}
$$

have the same law since $Y_{k}$ has Gaussian distribution $G_{k}$ with the same covariance matrix as $Z_{k}$ and thus as $P_{s_{k}} x_{1}$, by (3.23) and stationarity. For any integers $l, m, n$ let $F=F(l, m)$ denote the joint distribution of $\left\{x_{j}, 1 \leqslant j \leqslant l\right\}$ and $\left\{Y_{k}, 1 \leqslant k \leqslant\right.$ $m$ ) and let $G=G(m, n)$ be the joint distribution of

$$
\left\{\left(t_{k}-t_{k-1}\right)^{-1 / 2} P_{s_{k}}\left(X\left(t_{k}\right)-X\left(t_{k-1}\right)\right), 1 \leqslant k \leqslant m\right\} \quad \text { and } \quad\left\{X\left(r_{i}\right), 1 \leqslant i \leqslant n\right\}
$$

where $r_{i}$ are nonnegative numbers. Then by the above remarks the second marginal of $F$ equals the first marginal of $G$. Hence by Lemma Al of $[1$, p. 53], there is a probability space and three random elements $\xi_{i}, 1 \leqslant i \leqslant 3$, such that $\xi_{1}$ has the same distribution as $\left\{x_{j}, 1 \leqslant j \leqslant l\right\}, \xi_{2}$ has the same distribution as $\left\{Y_{k}, 1 \leqslant k \leqslant\right.$ $m\}$ or as $\left\{\left(t_{k}-t_{k-1}\right)^{-1 / 2} P_{s_{k}}\left(X\left(t_{k}\right)-X\left(t_{k-1}\right)\right), 1 \leqslant k \leqslant m\right\}$ and $\xi_{3}$ has the same distribution as $\left\{X\left(r_{i}\right), 1 \leqslant i \leqslant n\right\}$. As $l, m, n$ and $\left\{r_{i}, 1 \leqslant i \leqslant n\right\}$ vary this defines a consistent system $H$ of distributions $H(l, m, n)$. Hence by Kolmogorov's theorem there exists a probability space on which we can redefine the sequences $\left\{x_{j}, j \geqslant 1\right\}$ and $\left\{Y_{k}, k \geqslant 1\right\}$ without changing their joint law and on which there exists a Brownian motion $\{X(t), t \geqslant 0\}$ with mean zero and covariance function given by (1.1) such that

$$
\left(t_{k}-t_{k-1}\right)^{-1 / 2} P_{s_{k}}\left(X\left(t_{k}\right)-X\left(t_{k-1}\right)\right)=Y_{k}, \quad k \geqslant 1 .
$$

We now show that $\{X(t), t \geqslant 0\}$ has the desired properties. Let $t>0$ be given and define $m$ by $t_{m-1}<t \leqslant t_{m}$. Then

$$
\begin{aligned}
\sup _{t_{m-1}<t<t_{m}}\left\|\sum_{j<t} x_{j}-X(t)\right\| & \\
\leqslant & \sup _{t_{m-1}<t<t_{m}}\left\|\sum_{t_{m-1}<j<t} x_{j}\right\|+\sup _{t_{m-1}<t<t_{m}}\left\|X(t)-X\left(t_{m-1}\right)\right\| \\
& +\sum_{1<k<m}\left\|\sum_{t_{k-1}<j<t_{k}} P_{s_{k}} x_{j}-P_{s_{k}}\left(X\left(t_{k}\right)-X\left(t_{k-1}\right)\right)\right\| \\
& +\sum_{1<k<m}\left\|\sum_{t_{k-1}<j<t_{k}}\left(x_{j}-P_{s_{k}} x_{j}\right)\right\| \\
& +\sum_{1<k<m}\left\|X\left(t_{k}\right)-X\left(t_{k-1}\right)-P_{s_{k}}\left(X\left(t_{k}\right)-X\left(t_{k-1}\right)\right)\right\|,
\end{aligned}
$$

where we take $t_{0}=0$. We shall consider these terms one by one. Call them I, II, III, IV and V. By Lemmas 3.4 and 3.5

$$
\mathrm{I}+\mathrm{II} \ll u\left(s_{m}\right)^{\varepsilon / 2}\left(t_{m} \log \log t_{m}\right)^{1 / 2} \text { a.s. }
$$

By (3.23), (3.25), (3.26), (3.5) and (3.10)

$$
\text { III } \ll \sum_{k<m} t_{k}^{1 / 2-\lambda_{1}} \ll t_{m-1}^{1 / 2-\lambda_{1} / 2} \text { a.s. }
$$


By Lemmas 3.2, 3.3 and (3.8)

$$
\mathrm{IV}+\mathrm{V} \ll \sum_{k<m} u\left(s_{k}\right)^{3 \varepsilon / 2}\left(n_{k} \log \log t_{k}\right)^{1 / 2} \ll t_{m-1}^{1 / 2} u\left(s_{m-1}\right)^{\varepsilon} \quad \text { a.s. }
$$

It follows from (3.5) and (3.7) that $t_{m} \leqslant 2 t_{m-1}$. Also from (1.6) and (3.14) that $u\left(s_{m-1}\right) \leqslant 2 u\left(s_{m}\right)$. Thus we can extrapolate between $t_{m-1}$ and $t_{m}$ and $s_{m-1}$ and $s_{m}$ to get

$$
\left\|\sum_{j \leqslant t} x_{j}-X(t)\right\| \ll(u(\alpha / \log t))^{\varepsilon / 2}(t \log \log t)^{1 / 2} \text { a.s. }
$$

Here we used (3.4). It is easy to see that the dominant term in (3.31) is contributed by (3.28). Note also that (1.6) implies that

$$
u^{\varepsilon}(\alpha / \log t) \geqslant \alpha / \log t
$$

so that the bound in (3.29) is smaller than the bound in (3.28). The bound in (3.32) also justifies the argument following (3.16). This completes the proof of Case (i).

3.2. Case (ii). Here it is enough to simply choose $u_{1}\left(s_{k}\right)=s_{k}^{\beta}$. We define $s_{k}$ and $t_{k}$ as in (3.2) and (3.4) respectively. However, all the calculations are much simpler now because we get specific values for all quantities in terms of $k$. In particular

$$
s_{k}=(\alpha / k)^{1 /(1+\beta)} \text {. }
$$

We define $n_{k}$ as in (3.5) and given (3.33) it is simple to obtain

$$
n_{k} \sim \alpha^{\prime} t_{k} k^{-\beta /(1+\beta)}=\alpha^{\prime \prime} t_{k} u_{1}\left(s_{k}\right)
$$

for positive constants $\alpha^{\prime}, \alpha^{\prime \prime}$ depending on $\alpha$ and $\beta$. This gives us (3.7) for $n_{k}=n_{k, 1}$. The critical inequality (3.8) now is an easy consequence of (3.7) using integration by parts, i.e.

$$
\begin{aligned}
\sum_{k<m} n_{k}^{1 / 2} u_{1}\left(s_{k}\right)^{3 / 2} & \ll \sum_{k<m} t_{k}^{1 / 2} u_{1}^{2}\left(s_{k}\right) \ll \sum_{k<m} \exp \left(\frac{1}{2} \alpha k^{1 /(1+\beta)}\right) k^{-2 \beta /(1+\beta)} \\
& \ll \exp \left(\frac{1}{2} \alpha m^{1 /(1+\beta)}\right) m^{-\beta /(1+\beta)} \ll t_{m}^{1 / 2} u_{1}\left(s_{m}\right) .
\end{aligned}
$$

Analogues of (3.9) and (3.10) follow immediately.

Now that we have the basic inequalities of Lemma 3.1 everything goes through as in Case (i) with the sole exception that we must take $\rho=4\left((1+\beta) \log \log t_{k}\right)^{1 / 2}$ in Lemmas 3.2 and 3.3. We finally get (3.31) with $u(s)=s^{\beta}$. This completes the proof in Case (ii).

3.3. Case (iii). We define $s_{k}=k^{-1 / \beta}$ and $t_{k}=s_{k}^{-\alpha}=k^{\alpha / \beta}$. It is completely elementary to obtain

$$
n_{k} \sim C_{1} t_{k} k^{-1}=C_{2} t_{k} u\left(s_{k}\right)
$$

for some constants $C_{1}$ and $C_{2}$. Also

$$
\begin{aligned}
\sum_{k<m} n_{k} u_{1}\left(s_{k}\right)^{3 / 2} & \ll \sum_{k<m} t_{k}^{1 / 2} u_{1}^{2}\left(s_{k}\right) \ll \sum_{k<m} k^{\alpha /(2 \beta)-2} \\
& \ll m^{\alpha /(2 \beta)-1} \ll t_{m}^{1 / 2} u_{1}\left(s_{m}\right) .
\end{aligned}
$$


In place of inequality (3.9) we do the estimate of the Prohorov distance in (3.24) directly and get

$$
\pi\left(F_{k}, G_{k}\right) \ll n_{k}^{-\delta / 9} \cdot s_{k}^{-4 / 3} \ll k^{-3}=t_{k}^{-\lambda_{2}}, \quad \lambda_{2}>0,
$$

if $\alpha \geqslant(36 \beta+12) / \delta$.

As in Case (ii) we must redefine $\rho$ in Lemmas 3.2 and 3.3. We choose $\rho=$ $C_{3}\left(\log t_{k}\right)^{1 / 2}=C_{4}(\log k)^{1 / 2}$ where $C_{3}$ and $C_{4}$ are positive constants depending on $\alpha$ and $\beta$. It is easy to see that $\rho c \leqslant 1$. The term $\rho=C_{4}(\log k)^{1 / 2}$ can be absorbed into the $t_{k}^{-\lambda}$ term since $u\left(s_{k}\right)=k^{-1}$ and thus, by (3.35) $n_{k}$ is of the right order. The same is true for the terms in (3.36).

4. Processes on $\mathbf{C}(K, \tau)$. Let $(K, \tau)$ be a compact metric or pseudometric space and, as usual, let $\mathbf{C}(K)=\mathbf{C}(K, \tau)$ be the Banach space of continuous complex-valued functions in the supremum norm $\|\cdot\|_{\infty}$. By treating real and imaginary parts as pairs we still can apply Theorem 1.1. We begin by relating the expression in (1.4) to a uniform Lipschitz norm on $\mathbf{C}(K)$. For $\varepsilon>0$ let $N=N_{\tau}(s)$ denote the smallest number of open balls of radius $s$ in the $\tau$-metric or pseudometric, with centers in $K$, that covers $K$. Pick one such covering $\left\{U_{i}, i=1, \ldots, N\right\}$ and let $v_{i}$ denote the center of $U_{i}$. It is well known that there exists a partition of unity subordinated to $\left\{U_{i}\right\}$. That is, there exists a family of real valued functions $\left\{\phi_{i}, i=1, \ldots, N\right\}$ defined on $K$ such that $0 \leqslant \phi_{i} \leqslant 1, \sum_{i=1}^{N} \phi_{i}(y)=1$ for all $y \in K$ and the support of each $\phi_{i}$ is contained in $U_{i}$. Following $[4, \S 9]$ define, for $x \in \mathbf{C}(K)$,

$$
P_{s} x=\sum_{i=1}^{N} x\left(v_{i}\right) \phi_{i} \text {. }
$$

Clearly $\left\|P_{s}\right\|=1$ so $P_{s}$ is a bounded linear projection on $\mathrm{C}(K)$. The following lemma is taken from $[4, \S 11]$.

LEMMA 4.1. Let $Z$ be a $\mathbf{C}(K, \tau)$-valued random variable and $T(s)$ an integer-valued function. $T(s) \uparrow \infty$ as $s \downarrow 0$. Suppose

$$
E\left[\sup _{\substack{\tau\left(v, v^{\prime}\right)<s \\ v, v^{\prime} \in K}}\left|Z(v)-Z\left(v^{\prime}\right)\right|\right]=\psi(s) .
$$

Then there exists a projection $P_{s}: \mathbf{C}(K) \rightarrow \mathbf{C}(K)$ such that $\operatorname{dim} P_{s}=T(s)$ and such that

$$
E\left\|Z-P_{s} Z\right\| \leqslant \psi\left(N_{\tau}^{-1}(T(s))\right)
$$

where $N_{\tau}^{-1}$ is the inverse function of $N_{\tau}$.

Proof. We have

$$
\left(x-P_{s} x\right)(v)=\sum_{i=1}^{N}\left(x(v)-x\left(v_{i}\right)\right) \phi_{i}(v) .
$$

If $v \in K$, then $\phi_{i}(v)=0$ unless $\tau\left(v, v_{i}\right) \leqslant s$. Therefore

$$
\left\|x-P_{s} x\right\| \leqslant \sup _{\substack{\tau\left(v, v^{\prime}\right)<s \\ v, v^{\prime} \in K}}\left|x(v)-x\left(v^{\prime}\right)\right| .
$$


This together with (4.1) gives $E\left\|Z-P_{s} Z\right\| \leqslant \psi(s)$, where $\operatorname{dim} P_{s}=N_{\tau}(s)$. The inequality in (4.2) now results from a simple change of variables.

We shall now consider several processes for which we can obtain estimates of the function $u(s)$ in Theorem 1.1 and (1.4) via Lemma 4.1. Let $\left\{a_{k}, k \geqslant 1\right\} \in l^{2}$ be complex numbers, $\left\{\varepsilon_{k}, k \geqslant 1\right\}$ a Rademacher sequence (i.e. a sequence of independent random variables with $\left.P\left(\varepsilon_{k}=1\right)=P\left(\varepsilon_{k}=-1\right)=\frac{1}{2}\right)$ and $\left\{\xi_{k}, k \geqslant 1\right\}$ a sequence of complex-valued random variables such that

$$
\sup _{k} E^{1 / 2}\left|\xi_{k}\right|^{2} \leqslant Q
$$

The random variables $\xi_{k}$ need not be independent, but the sequences $\left\{\xi_{k}, k \geqslant 1\right\}$ and $\left\{\varepsilon_{k}, k \geqslant 1\right\}$ are assumed to be independent of each other.

(1) Random Fourier series on a locally compact abelian group $G$. Let $\Gamma$ denote the character group of $G$ and let $K$ be a compact symmetric neighborhood of the identity of $G$. We will assume that $\Gamma$ is countable and hence that $K$ is metrizable. Let $\left\{\gamma_{k}, k \geqslant 1\right\}$ be some ordering of the elements of $\Gamma$ and let $\tau$ be a metric on $K$. We consider the $C(K, \tau)$-valued random variables

$$
x_{1}(v)=\sum_{k>1} a_{k} \varepsilon_{k} \xi_{k} \gamma_{k}(v), \quad v \in K
$$

and define a translation invariant pseudometric on $G$

$$
\sigma_{1}\left(v, v^{\prime}\right)=\left(\sum_{k>1}\left|a_{k}\right|^{2}\left|\gamma_{k}\left(v-v^{\prime}\right)-1\right|^{2}\right)^{1 / 2} .
$$

Note that if $v, v^{\prime} \in K$ the domain of $\sigma_{1}$ is $K \oplus K$.

(2) Uniformly Lipschitz continuous stochastic processes. Let $(K, \tau)$ be a compact metric space and let $\left\{x_{2}(v), v \in K\right\}$ be a complex-valued stochastic process on the probability space $(\Omega, F, P)$ such that $E x_{2}(v)=$ constant and such that $E\left|x\left(v_{0}\right)\right|^{2}<$ $\infty$ for some $v_{0} \in K$. Let $\sigma_{2}\left(v, v^{\prime}\right)$ be a translation invariant pseudometric on $K$, continuous with respect to $\tau$ such that for all $v, v^{\prime} \in K$,

$$
\left|x_{2}(v)-x_{2}\left(v^{\prime}\right)\right| \leqslant M \sigma_{2}\left(v, v^{\prime}\right) \text {, }
$$

where $M$ is a random variable on $(\Omega, F, P)$ satisfying $E M^{2}<\infty$.

The next two examples are complex-valued stochastic processes on $\mathbf{R}^{N}$. We continue to use the notation $\mathbf{C}(K, \tau)$, but now $K=\left[-\frac{1}{2}, \frac{1}{2}\right]^{N}$ and $\tau$ is the ordinary Euclidean metric. Let $\left\{\lambda_{k}, k \geqslant 1\right\}$ be a sequence of random variables with values in $\mathbf{R}^{N}$.

(3) Symmetrized empirical characteristic processes. Assume that the sequence $\left\{\xi_{k}, \lambda_{k}, k \geqslant 1\right\}$ is independent of $\left\{\varepsilon_{k}, k \geqslant 1\right\}$. The sequences $\left\{\xi_{k}, k \geqslant 1\right\}$ and $\left\{\lambda_{k}\right.$, $k \geqslant 1\}$ need not be sequences of independent random variables nor do the sequences have to be independent of each other. Define

$$
x_{3}(v)=\sum_{k>1} a_{k} \varepsilon_{k} \xi_{k} e^{1\left\langle\lambda_{k}, v\right\rangle}, \quad v \in K
$$

Let

$$
\phi_{k}(v)=2\left(E\left|\xi_{k}\right|^{2} \sin ^{2} \frac{1}{2}\left\langle\lambda_{k}, v\right\rangle\right)^{1 / 2}, \quad v \in[-1,1]^{N},
$$


and define the translation-invariant metric or pseudometric

$$
\sigma_{3}\left(v, v^{\prime}\right)=\left(\sum_{k>1}\left|a_{k}\right|^{2} \phi_{k}^{2}\left(v-v^{\prime}\right)\right)^{1 / 2} .
$$

(4) Empirical characteristic process. We now assume that $\left\{\left(\xi_{k}, \lambda_{k}\right), k \geqslant 1\right\}$ is a sequence of independent random variables $\left(\left\{\xi_{k}, k \geqslant 1\right\}\right.$ and $\left\{\lambda_{k}, k \geqslant 1\right\}$ need not be independent of each other). Let

$$
c_{k}(v)=E \xi_{k} e^{i\left\langle\lambda_{k}, v\right\rangle}, \quad v \in K
$$

We define

$$
x_{4}(v)=\sum_{k>1} a_{k}\left(\xi_{k} e^{i\left\langle\lambda_{k}, v\right\rangle}-c_{k}(v)\right), \quad v \in K
$$

and $\sigma_{4}$ the same way as $\sigma_{3}$ given in (4.9).

Let $\left\{x_{i j}, j \geqslant 1\right\}$ be a sequence of independent copies of $x_{i}, i \leqslant i \leqslant 4$, and define

$$
S_{i n}=\sum_{j<n} x_{i j}
$$

Furthermore, for all these processes we assume

$$
\int_{0}^{\infty}\left(\log N_{\sigma_{i}}(K, \varepsilon)\right)^{1 / 2} d \varepsilon<\infty, \quad 1 \leqslant i<4
$$

TheOREM 4.2. Let $x_{i}, 1 \leqslant i \leqslant 4$, be the stochastic processes defined above. Assume that (4.13) holds and let $S_{\text {in }}$ be as defined in (4.12). Then for $1 \leqslant i \leqslant 4$,

$$
\begin{aligned}
\sup _{n>1} n^{-1 / 2} E\left\{\sup _{\tau\left(v, v^{\prime}\right)<s}\right. & \left.\left|S_{i n}(v)-S_{i n}\left(v^{\prime}\right)\right|\right\} \\
\leqslant & \leqslant\left(\int_{0}^{\hat{\sigma}_{i}(s)}\left(\log N_{\sigma_{i}}(K, \varepsilon)\right)^{1 / 2} d \varepsilon+\hat{\sigma}_{i}(s)\right)=\psi_{i}(s),
\end{aligned}
$$

where $C$ is a finite positive constant and

$$
\begin{gathered}
\hat{\sigma}_{1}(s)=E^{1 / 2}\left\{\sup _{\tau\left(v, v^{\prime}\right) \leqslant s} \sum_{k>1}\left|a_{k}\right|^{2}\left|\xi_{k}\right|^{2}\left|\gamma_{k}\left(v-v^{\prime}\right)-1\right|^{2}\right\}, \\
\hat{\sigma}_{2}(s)=\sup _{\tau\left(v, v^{\prime}\right) \leqslant s} \sigma_{2}\left(v, v^{\prime}\right),
\end{gathered}
$$

and, for $i=3,4$,

$$
\hat{\sigma}_{i}(s)=2 E^{1 / 2}\left\{\sup _{\tau\left(v, v^{\prime}\right)<s} \sum_{k>1}\left|a_{k}\right|^{2}\left|\xi_{k}\right|^{2} \sin ^{2}\left\langle\lambda_{k}, v-v^{\prime}\right\rangle\right\} .
$$

Furthermore, $\lim _{s \rightarrow 0} \hat{\sigma}_{i}(s)=0,1 \leqslant i \leqslant 4$, and this together with (4.14) gives $\lim _{s \rightarrow 0} \psi_{i}(s)=0,1 \leqslant i \leqslant 4$.

Proof. The proof for $i=1$ follows immediately from Theorem 1.4, Chapter 3, of [16] as explained in the proof of Theorem 1.1, Chapter 4, of [16].

The proof for $i=3$ is the same as that for $i=1$ except that the expectation (see (1.23), Chapter 3, of [16]) must also be taken with respect to $\left\{\lambda_{k}\right\}$. We will outline the beginning of the proof. Let $\left\{\varepsilon_{k}\right\}$ be defined on the probability space $\left(\Omega_{2}, F_{2}, P_{2}\right)$ and $\left\{\xi_{k}, \lambda_{k}\right\}$ on $\left(\Omega_{1}, F_{1}, P_{1}\right)$ and let $E_{2}$ and $E_{1}$ be the corresponding expectation 
operators. Let $(\Omega, F, P)$ denote the product probability space $\left(\Omega_{1} \times \Omega_{2}, F_{1} \times F_{2}\right.$, $P_{1} \times P_{2}$ ) and denote the expectation operator on this space by $E$. It is obvious that

$$
\sigma_{3}\left(v-v^{\prime}\right)=n^{-1 / 2} E^{1 / 2}\left|S_{3 n}(v)-S_{3 n}\left(v^{\prime}\right)\right|^{2} .
$$

Consider, for fixed $\omega_{1} \in \Omega_{1}$

$$
n^{-1 / 2} S_{3 n}\left(v, \omega_{1}\right)=\sum_{k>1} a_{k} n^{-1 / 2} \sum_{j<n} \varepsilon_{k j} \xi_{k j}\left(\omega_{1}\right) e^{i\left\langle\lambda_{w}\left(\omega_{1}\right), v\right\rangle}
$$

and

$$
\begin{aligned}
\sigma_{3 n}\left(v-v^{\prime}, \omega_{1}\right) & =n^{-1 / 2} E_{2}^{1 / 2}\left|S_{3 n}\left(v, \omega_{1}\right)-S_{3 n}\left(v^{\prime}, \omega_{1}\right)\right|^{2} \\
& =2\left(\sum_{k>1}\left|a_{k}\right|^{2}\left|\xi_{k}\left(\omega_{1}\right)\right|^{2} \sin ^{2} \frac{1}{2}\left\langle\lambda_{k}\left(\omega_{1}\right), v-v^{\prime}\right\rangle\right)^{1 / 2} .
\end{aligned}
$$

Note that $\sigma_{3 n}=\sigma_{31}$ for all $n$. Let

$$
\hat{\sigma}_{3}\left(s, \omega_{1}\right)=\sup _{\tau\left(v, v^{\prime}\right)<s} \sigma_{3 n}\left(v-v^{\prime}, \omega_{1}\right) \text { and } \hat{\sigma}_{3}(s)=E_{1}^{1 / 2}\left(\hat{\sigma}_{3}\left(s, \omega_{1}\right)\right)^{2} .
$$

Following the proof of Theorem 1.4, Chapter 3, of [16] we have

$$
\begin{aligned}
E_{2} \sup _{\tau\left(v, v^{\prime}\right)<s} \mid S_{3 n}\left(v, \omega_{1}\right) & -S_{3 n}\left(v^{\prime}, \omega_{1}\right) \mid \\
& \leqslant E_{2} \sup _{\sigma_{3 n}\left(v-v^{\prime}, \omega_{1}\right)<\hat{\sigma}_{3 n}\left(s, \omega_{1}\right)}\left|S_{3 n}\left(v, \omega_{1}\right)-S_{3 n}\left(v^{\prime}, \omega_{1}\right)\right| .
\end{aligned}
$$

Note that $S_{3 n}\left(v, \omega_{1}\right)$ is a subgaussian process. Therefore we can continue the proof with the third inequality in (1.21), Chapter 3 , of [16], with the minor exception that $g(a)$ in that paper is now defined as $E_{1}^{1 / 2}\left(\hat{\sigma}_{3}\left(a, \omega_{1}\right)\right)^{2}$ instead of $E_{1} \hat{\sigma}_{3}\left(a, \omega_{1}\right)$.

The case $i=4$ follows immediately from the case $i=3$ since by (10) of [15]

$$
E \sup _{\tau\left(v, v^{\prime}\right)<s}\left|S_{4 n}(v)-S_{4 n}\left(v^{\prime}\right)\right| \leqslant 2 E \sup _{\tau\left(v, v^{\prime}\right)<s}\left|S_{3 n}(v)-S_{3 n}\left(v^{\prime}\right)\right| .
$$

Lastly we consider the case $i=2$. The proof here is much easier than in the other cases. It follows from Theorem 3.1, Chapter 2, of [16], which is a generalization of a familiar theorem of Dudley. The idea, in the symmetric case, is to consider

$$
\tilde{S}_{n}\left(v, \omega_{1}\right)=\sum_{j<n} \varepsilon_{k} x_{2 j}\left(v, \omega_{1}\right) / \sum_{j<n} M_{j}^{2}\left(\omega_{1}\right)
$$

as a subgaussian process. This satisfies

$$
E_{2}^{1 / 2}\left|\tilde{S}_{n}\left(v, \omega_{1}\right)-\tilde{S}_{n}\left(v^{\prime}, \omega_{1}\right)\right|^{2} \leqslant \sigma_{2}\left(v, v^{\prime}\right) .
$$

Also we have

$$
\begin{aligned}
E_{2} \sup _{\tau\left(v, v^{\prime}\right)<s}\left|\tilde{S}_{n}\left(v, \omega_{1}\right)-\tilde{S}_{n}\left(v^{\prime}, \omega_{1}\right)\right| \\
\quad \leqslant E \sup _{\sigma_{2}\left(v, v^{\prime}\right)<\hat{\sigma}_{2}(s)}\left|\tilde{S}_{n}\left(v, \omega_{1}\right)-\tilde{S}_{n}\left(v^{\prime}, \omega_{1}\right)\right| .
\end{aligned}
$$


The desired result, (4.14), now follows by applying Theorem 3.1, Chapter 2, of [16], to (4.18) and then applying the expectation operator $E_{1}$. To desymmetrize we use Lemma 2 of [15].

Finally the fact that $\lim _{s \rightarrow 0} \hat{\sigma}_{i}(s)=0$ follows from the dominated convergence theorem for $i=1,3,4$ and by hypothesis in the case $i=2$.

RemarK 4.3. It is easy to see that $\sup _{v \in K} E\left|x_{i}(v)\right|^{2}<\infty, 1 \leqslant i \leqslant 4$. This imples that for any $v_{1}, \ldots, v_{m} \in K$ the vector $\left(x_{i}\left(v_{1}\right), \ldots, x_{i}\left(v_{m}\right)\right)$ satisfies the central limit theorem on $\mathbf{R}^{m}, 1 \leqslant i \leqslant 4$. This together with Theorem 4.2 shows that the processes $x_{i}, 1 \leqslant i \leqslant 4$, satisfy the central limit theorem on $\mathbf{C}(K, \tau)$ for the appropriate space $(K, \tau)$. For the processes in (1) this result was obtained in [14] and extended in [16], (see Theorem 1.1, Chapter 4, of [16]) and for the processes in (2) it was obtained in [8]. For certain processes in (3) and (4) it was obtained in [15] and, in general, as we saw above, the central limit theorem for the processes in (3) and (4) is an immediate corollary of Theorem 1.4, Chapter 3, of [16]. It is interesting to note that in the cases $i=1,3,4$, the limiting Gaussian measure exists only if certain stationary Gaussian processes exist and these exist if and only if (4.13) holds. Thus, for the processes of examples (1), (3) and (4) condition (4.13) is necessary and sufficient for the central limit theorem.

Lemma 4.1 and Theorem 4.2 enable us to apply Theorem 1.1 to the four classes of stochastic processes considered here. In the remainder of this section we will introduce various simplifying assumptions that enable us to make more explicit computations and we will give a proof of Theorem 1.2.

In all that follows in this section we will restrict our attention to the case $K=\left[-\frac{1}{2}, \frac{1}{2}\right]^{N}$ with metric $\tau\left(v, v^{\prime}\right)=\left|v-v^{\prime}\right|$. We will obtain an upper bound for the integral in (4.12) when $\sigma_{i}$ is translation invariant. Of course, for the processes in (1), (3) and (4) $\sigma_{i}$ is translation invariant and for the processes in (2) this is an extra hypothesis.

Now let $\sigma\left(v, v^{\prime}\right)$ be a translation invariant metric on $K$. For notational convenience we write $\sigma\left(v, v^{\prime}\right)=\sigma\left(0, v-v^{\prime}\right)=\sigma\left(v-v^{\prime}\right)$. We define

$$
m_{\sigma}(y)=\lambda\left\{z \in[-1,1]^{N}: \sigma(z)<y\right\}
$$

where $\lambda$ is Lebesgue measure.

Lemma 4.4. Let $K=\left[-\frac{1}{2}, \frac{1}{2}\right]^{N}$ and let $\sigma$ be a translation invariant metric on $K$. Write

$$
\hat{\sigma}(s)=\sup _{|u|<s} \sigma(u)
$$

Then for $0<s \leqslant \frac{1}{2}$ we have

$$
\int_{0}^{\hat{\sigma}(s)}\left(\log N_{\sigma}(K, \varepsilon)\right)^{1 / 2} d \varepsilon \leqslant C\left[\hat{\sigma}(s)(\log 1 / s)^{1 / 2}+\int_{0}^{s} \frac{\hat{\sigma}(u)}{u(\log 1 / u)^{1 / 2}} d u\right]
$$

where $C$ is a constant depending on the dimension $N$.

Proof. By (1.2) and (1.9), Chapter 2, of [16] we have

$$
N_{\sigma}(K, \varepsilon) \leqslant 4^{N} / m_{\sigma}\left(\frac{1}{4} \varepsilon\right) \text {. }
$$


Therefore,

$$
\int_{0}^{\hat{\sigma}(s)}\left(\log N_{\sigma}(K, \varepsilon)\right)^{1 / 2} d \varepsilon \leqslant 4 \int_{0}^{s}\left(\log \frac{4^{N}}{m_{\sigma}(\hat{\sigma}(v))}\right)^{1 / 2} d \hat{\sigma}(v) .
$$

By (4.19) and (4.20) $m_{\sigma}(\hat{\sigma}(v)) \geqslant \lambda\left\{u \in[-1,1]^{N}:|u| \leqslant v\right\}=B_{N} v^{N}$ for $0 \leqslant v \leqslant \frac{1}{2}$, where $B_{N}$ is a constant depending on $N$. Using this in (4.22) we obtain (4.21).

REMARK 4.5. In order to use this lemma on the four types of processes considered we need to check that $\sup _{|u| \leqslant s} \sigma_{i}(u)=\hat{\sigma}_{i}(s)$. For $i=4$ this follows from (4.8), (4.9) and (4.17). The case $i=3$ is identical, the case $i=2$ is trivial and the case $i=1$ is equally simple.

Lemma 4.6. Let $K=\left[-\frac{1}{2}, \frac{1}{2}\right]^{N}$. Consider the processes $x_{3}$ and $x_{4}$ with the further assumption that the sequences $\left\{\xi_{k}\right\}$ and $\left\{\lambda_{k}\right\}$ are independent of each other. Let

$$
R_{k}(x)=P\left(\left|\lambda_{k}\right| \leqslant x\right)
$$

Then for $i=3$ and 4 ,

$$
\hat{\sigma}_{i}(s) \leqslant Q\left(\sum_{k>1}\left|a_{k}\right|^{2} G_{k}(s)\right)^{1 / 2}
$$

where $Q$ is given in (4.3) and

$$
G_{k}(s)=2 s^{2} \int_{0}^{1 / s} x\left(1-R_{k}(x)\right) d x
$$

Proof. By (4.17) for $i=3,4$,

$$
\begin{aligned}
\hat{\sigma}_{i}^{2}(s) & =4 E \sup _{|u|<s} \sum_{k>1}\left|a_{k}\right|^{2}\left|\xi_{k}\right|^{2} \sin ^{2} \frac{1}{2}\left\langle\lambda_{k}, u\right\rangle \\
& \leqslant E \sum_{k>1}\left|a_{k}\right|^{2}\left|\xi_{k}\right|^{2}\left(\left|\lambda_{k}\right|^{2} s^{2} \wedge 1\right) \leqslant Q \sum_{k>1}\left|a_{k}\right|^{2} E\left(\left|\lambda_{k}\right|^{2} s^{2} \wedge 1\right) .
\end{aligned}
$$

But by a simple integration by parts

$$
E\left(\left|\lambda_{k}\right|^{2} s^{2} \wedge 1\right)=s^{2} \int_{0}^{1 / s} x^{2} d R_{k}(x)-\left(1-R_{k}(1 / s)\right)=G_{k}(x) .
$$

COROLlaRy 4.7. In particular, if in the case $i=3$ the random vectors $\lambda_{k}$ are constants

$$
x_{3}(v)=x_{1}(v)=\sum_{k>1} a_{k} \varepsilon_{k} \xi_{k} e^{i\left\langle\lambda_{k}, v\right\rangle}, \quad v \in K
$$

is a random Fourier series and we have

$$
\hat{\sigma}_{3}(s)=\hat{\sigma}_{1}(s) \leqslant Q\left(2 s^{2} \int_{0}^{1 / s} v(1-T(v)) d v\right)^{1 / 2}
$$

where

$$
T(v)=\sum_{\left|\lambda_{k}\right|<v}\left|a_{k}\right|^{2}
$$


Proof. This can be obtained directly from Lemma 4.6, but easier still from the last line of (4.25) since

$$
\begin{aligned}
\sum_{k>1}\left|a_{k}\right|^{2}\left(\left|\lambda_{k}\right|^{2} s^{2} \wedge 1\right) & =s^{2} \sum_{\left|\lambda_{k}\right| s<1}\left|a_{k}\right|^{2}\left|\lambda_{k}\right|^{2}+\sum_{\left|\lambda_{k}\right| s>1}\left|a_{k}\right|^{2} \\
& =s^{2} \int_{0}^{1 / s} v^{2} d T(v)-(1-T(1 / s))=2 s^{2} \int_{0}^{1 / s} v(1-T(v)) d v .
\end{aligned}
$$

Proof of TheOrem 1.2. We apply the above results to the random variable $y(v)=\exp (i\langle X, v\rangle)-c(v), v \in K$, where $X$ is a random variable with values in $\mathbf{R}^{N}$ and $c(v)$ is its characteristic function. This is a special case of the processes given in (4). Since $y$ is a bounded random variable we will take $\varepsilon=\frac{1}{4}$ in (1.5). Recall that $(K, \tau)$ is $\left[-\frac{1}{2}, \frac{1}{2}\right]^{N}$ with the Euclidean metric. Thus we have $N_{\tau}(s)>$ $\left(B_{N} / s\right)^{N}$ for some constant $B_{N}$ depending only upon $N$. Therefore $N_{\tau}^{-1}(s) \leqslant$ $B_{N} s^{-1 / N}$. In Theorem 1.1(i) $\operatorname{dim} P_{s}=\exp (1 / s)$. Therefore by Lemma 4.1 and Theorem 4.2 with $i=4$ we obtain for all $n \geqslant 1$,

$$
n^{-1 / 2} E\left\|S_{n}-P_{s} S_{n}\right\| \leqslant \psi_{4}\left(B_{N} \exp (-1 /(N s))\right) \text {. }
$$

Let $w$ be a concave majorant of $\psi_{4}^{1 / 4}$ such that $\lim _{s \rightarrow 0} w(s)=0$. Such a function $w$ exists since by Theorem $4.2 \lim _{s \rightarrow 0} \psi_{4}(s)=0$. Then by (1.7) and (1.8) and since $\varepsilon=\frac{1}{4}$ is an admissible choice we obtain (1.22). (The functions $\psi$ in (1.21) and $\psi_{4}$ in (4.14) are the same.)

To prove the remainder of Theorem 1.2 we first observe that by (4) the metric associated with the process $y$, as defined above, is translation invariant and given by

$$
\sigma_{4}(v)=\sigma_{4}(0, v)=2\left(E \sin ^{2} \frac{1}{2}\langle X, u\rangle\right)^{1 / 2}
$$

and that by Lemma 4.6

$$
\hat{\sigma}_{4}(s)=\left(2 s^{2} \int_{0}^{1 / s} x(1-R(x)) d x\right)^{1 / 2}
$$

where $R(x)=P(|X| \leqslant x)$. Hence (1.23) implies

$$
\hat{\sigma}_{4}(s) \ll(\log 1 / s)^{-1 / 2}\left(\log \log \frac{1}{s}\right)^{-8}, \quad s \rightarrow 0,
$$

and thus by (4.14) and (4.21)

$$
\psi_{4}(s) \ll(\log \log 1 / s)^{-g+1} .
$$

Similarly (1.25) implies

$$
\psi_{4}(s) \ll(\log 1 / s)^{-(g-1) / 2}
$$

and (1.27) implies

$$
\psi_{4}(s) \ll s^{g / 2}(\log 1 / s)^{1 / 2} .
$$

Hence if (1.23) holds, (1.24) follows directly from (1.8), (4.29) and (4.30). In the same way (1.25) implies (1.26). Finally, if (1.27) holds we have $\operatorname{dim} P(s)=1 / s$ and by Lemma 4.1 ,

$$
n^{-1 / 2} E\left\|S_{n}-P_{s} S_{n}\right\| \leqslant \psi_{4}\left(B_{N} s^{1 / N}\right) .
$$

(1.28) follows now from (1.10) and (4.33). 
REMARK 4.8. From the proof of Theorem 1.2 it is clear that it also holds for the sequence $\left\{\varepsilon_{j} \exp \left(i\left\langle X_{j}, v\right\rangle\right), j \geqslant 1, v \in\left[-\frac{1}{2}, \frac{1}{2}\right]^{N}\right\}$ where $X_{j}$ are independent, identically distributed copies of the $\mathbf{R}^{N}$-valued random variable $X$. This is because the functions $R$ and $\sigma$ are the same for this process. In this case the approximating Gaussian process is $\left\{H_{1}(v, t), v \in\left[-\frac{1}{2}, \frac{1}{2}\right]^{N}, t \geqslant 0\right\}$ with mean zero and covariance function

$$
E\left\{H_{1}(v, t) \overline{H_{1}\left(v^{\prime}, t^{\prime}\right)}\right\}=\min \left(t, t^{\prime}\right) c\left(v-v^{\prime}\right) .
$$

Similarly, Theorem 1.2 remains valid for the partial sum process of independent copies of the process considered in (4.26) if $R(x)$ in Theorem 1.2 is replaced by $T(x)$ given in (4.28). In this case the approximating Gaussian process is $\left\{H_{2}(v, t)\right.$, $\left.v \in\left[-\frac{1}{2}, \frac{1}{2}\right]^{N}, t \geqslant 0\right\}$ with mean zero and covariance function

$$
E\left\{H_{2}(v, t) \overline{H_{2}\left(v^{\prime}, t^{\prime}\right)}\right\}=\min \left(t, t^{\prime}\right) \sum_{k>1}\left|a_{k}\right|^{2} E\left|\xi_{k}\right|^{2} e^{i\left\langle\lambda_{k}, v-v^{\prime}\right\rangle} .
$$

Also in this case we need $E\left\|x_{1}\right\|^{2+\delta}<\infty$. (See the next remark.)

REMARK 4.9. Theorem 1.1 requires that $E\left\|x_{1}\right\|^{2+\delta}<\infty$. We shall consider this condition for the four examples of processes given at the beginning of this section. For the processes in (2) it is easy to see that $E\left|x_{2}\left(v_{0}\right)\right|^{2+\delta}<\infty$ for some $v_{0} \in K$ and $E|M|^{2+\delta}<\infty$ is sufficient. For the processes in (1), (3) and (4) assume that $\left\{\left(\xi_{k}, \lambda_{k}\right), k \geqslant 1\right\}$ is a sequence of independent random variables. Then by Theorem 3.3 of [9] and (4.13) we have $E\left\|x_{i}\right\|^{2+\delta}<\infty$ if and only if $\sup _{k \geqslant 1} E\left|\xi_{k}\right|^{2+\delta}<\infty$, $i=1,3,4$.

In the general case of the processes given in (1) it is again true that if (4.13) holds then $E\left\|x_{1}\right\|^{2+\delta}<\infty$ if and only if $\sup _{k \geq 1} E\left|\xi_{k}\right|^{2+\delta}<\infty$. This is proved by following the proof of Theorem 1.1, Chapter 3, of [16] but with

$$
\|\cdot\|_{\Omega}=\left(E|\cdot|^{2+\delta}\right)^{1 /(2+\delta)}
$$

in (1.7). Also Corollary 4.6, Chapter 2, of [16] must be used in (1.3) of [16]. Finally, this same method can be used for the processes in (3) when $\left\{\left(\xi_{k}, \lambda_{k}\right), k \geqslant 1\right\}$ is not a sequence of independent random variables. However, our approach requires additional hypotheses besides (4.13) and $\sup _{k \geqslant 1} E\left|\xi_{k}\right|^{2+\delta}<\infty$. We will not go into the details here.

REMARK 4.10. These methods can also be used on the second order stochastic integrals considered by Fernique [5]. Theorems 4.2 and 1.2 can also be given for these processes.

5. Further applications. Let $\left\{x_{j}, j \geqslant 1\right\}$ be a sequence of independent identically distributed $B$-valued random variables, with mean zero, finite second moment and satisfying the central limit theorem. Then, according to a theorem of Pisier [19], $\left\{x_{j}, j \geqslant 1\right\}$ also satisfies the compact law of the iterated logarithm with cluster set determined by the covariance function $E\left\{f\left(x_{1}\right) g\left(x_{1}\right)\right\}, f, g \in B^{*}$. But this also follows from (1.2) and (1.1) since, as is well known Brownian motion satisfies the compact law of the iterated logarithm with cluster set determined by $E\{f(X(1)) g(X(1))\}, f, g \in B^{*}$. 
Upper and lower class refinements of the law of the iterated logarithm can easily be obtained from corresponding results for $B$-valued Brownian motion provided that in relation (1.7) the function $g$ satisfies

$$
g(t, \delta) \ll 1 / \log \log t .
$$

Indeed, consider Theorem 2.3 of Kuelbs [11] which is such an upper and lower class result for Brownian motion. It involves the integral

$$
\int^{\infty} \frac{(\phi(t))^{n_{1}}}{t} e^{-\phi^{2}(t) / 2} d t
$$

where $n_{1}$ is some finite integer. As noted in (2.5) of [11] in the proof of Theorem 2.3 of [11] there is no loss of generality to assume $(\log \log t)^{1 / 2} \leqslant \phi(t) \leqslant 2(\log \log t)^{1 / 2}$ for all sufficiently large $t$. Hence the convergence or divergence of the integral (5.2) is not affected if a function of size $\ll(\log \log t)^{-1 / 2}$ is added to $\phi(t)$. But if $(5.1)$ holds then (1.7) becomes

$$
\left\|\sum_{j<t} x_{j}-X(t)\right\| \ll(t / \log \log t)^{1 / 2} \text { a.s. }
$$

Consequently, Theorem 2.3 of Kuelbs [11] continues to hold for the partial sum process.

Another application deals with the simulation of the sample paths of Gaussian processes. Let $Y(v), v \in\left[-\frac{1}{2}, \frac{1}{2}\right]^{N}$, be a Gaussian process with spectral representation $\int_{\mathbf{R}^{N}} e^{i\langle v, \lambda\rangle} d b(F(\lambda))$ where $b$ is Brownian motion and $F$ is a distribution function on $\mathbf{R}^{N}$ called the spectrum of $Y$. Let $\left\{Y_{j}, j \geqslant 1\right\}$ be a sequence of independent copies of $Y$. Let $\left\{X_{j}, j \geqslant 1\right\}$ be a sequence of independent identically distributed $\mathbf{R}^{N}$-valued random variables with distribution function $F$. It follows from Theorem 1.1 and Remark 4.8 that with probability 1

$$
\sup _{v \in K}\left|n^{-1 / 2} \sum_{j<n} \varepsilon_{j} e^{i\left\langle X_{j}, v\right\rangle}-n^{-1 / 2} \sum_{j<n} Y_{j}\right| \ll g(n, \delta)(\log \log n)^{1 / 2}
$$

where $K=\left[-\frac{1}{2}, \frac{1}{2}\right]^{N}$. According to conditions on $R(x)=P\{|X| \leqslant x\}$ the term on the right in (5.3) will go to zero at various rates. Since $n^{-1 / 2} \sum_{j<n} Y_{j}$ is a process equal in law to $Y$ this suggests the possibility of simulating a path of $Y(v)$ by

$$
n^{-1 / 2} \sum_{j<n} \varepsilon_{j} e^{i\left\langle X_{j}, v\right\rangle}, \quad v \in K .
$$

The point is that it is probably easier to "generate" the sequence $\left\{X_{j}, j \geqslant 1\right\}$ with distribution function $F$ and to form (5.4) than to create sample paths of a Gaussian process with spectrum $F$. The term on the right in (5.3) tells how good the approximation is. Actually, what is perhaps even more desirable is an estimate of the form

$$
P\left\{\sup _{v \in K}\left|n^{-1 / 2} \sum_{j<n} e^{i\left\langle X_{j}, v\right\rangle}-Y(v)\right| \geqslant \varepsilon\right\} \leqslant h(n, \varepsilon)
$$

where $h(n, \varepsilon) \rightarrow 0$ as $n \rightarrow \infty$ for each $\varepsilon>0$. This can easily be obtained from our methods, by using the tail estimates we give before we apply the Borel Cantelli lemmas. 
We are grateful to Herold Dehling for his many helpful comments, in particular for suggesting that we formulate Theorem 1.1 in greater generality than $\mathbf{C}(K)$.

\section{REFERENCES}

1. István Berkes and Walter Philipp, Approximation theorems for independent and weakly dependent random vectors, Ann. Probab. 7 (1979), 29-54.

2. Leo Breiman, Probability, Addison-Wesley, Reading, Mass., 1968.

3. Sandor Csörgö, Limit behaviour of the empirical characteristic function, Ann. Probab. 8 (1981).

4. Herold Dehling, Limit theorems for sums of weakly dependent Banach space valued random variables, (1980) (preprint).

5. X. Fernique, Continuité et théorème centrale limite pour les transformes de Fourier des mesures aléatoire du second ordre, Z. Wahrsch. Verw. Gebiete 42 (1978), 57-68.

6. E. Gine and M. B. Marcus, On the central limit theorem in $C(k)$, Colloq. Statistical and Dynamical Properties of Gaussian Processes, Lecture Notes in Math., Springer-Verlag, New York, 1980.

7. J. Hoffmann-Jorgensen, Probability in Banach space, Lecture Notes in Math., vol. 598, SpringerVerlag, New York, 1977, pp. 1-186.

8. Naresh C. Jain and Michael B. Marcus, Central limit theorem for $\mathrm{C}(S)$-valued random variables, J. Funct. Anal. 19 (1975), 216-231.

9. Integrability of infinite sums of independent vector-valued random variables, Trans. Amer. Math. Soc. 212 (1975), 1-36.

10. _ Continuity of subgaussian processes, Advances in Probability, Vol. 4, Dekker, New York, 1978.

11. J. Kuelbs, Sample path behavior for Brownian motion in Banach spaces, Ann. Probab. 3 (1975), $247-261$.

12. ___ Kolmogorov's law of the iterated logarithm for Banach space valued random variables, Illinois J. Math. 21 (1977), 784-800.

13. J. Kuelbs and Walter Philipp, Almost sure invariance principles for partial sums of mixing B-valued random variables, Ann. Probab. 8 (1980), 1003-1036.

14. Michael B. Marcus, Continuity and the central limit theorem for random trigonometric series, $Z$. Wahrsch. Verw. Gebiete 42 (1978), 35-56.

15. Weak convergence of the empirical characteristic function, Ann. Probab. 8 (1981).

16. Michael B. Marcus and Gilles Pisier, Random Fourier series with applications to harmonic analysis, Ann. of Math. Studies, Princeton, Univ. Press, Princeton, N.J., 1981.

17. Walter Philipp, Almost sure invariance principles for sums of B-valued random variables, Lecture Notes in Math., vol. 709, Springer-Verlag, Berlin and New York, 1979, pp. 171-193.

18. Weak and $L^{p}$-invariance principles for sums of $B$-valued random variables, Ann. Probab. 8 (1980), 68-82.

19. G. Pisier, Le théorème de la limite centrale et la loi du logarithme iteré dans les espaces de Banach, Séminaire Maurey-Schwartz 1975-76, Exposé IV, L'Ecole Polytéchnique, Paris, 1975.

20. V. V. Yurinskii, On the error of the Gaussian approximation for convolutions, Theory Probab. Appl. 22 (1977), 236-247.

Department of Mathematics, NorthWestern University, Evanston, Iluinois 60201

Department of Mathematics, University of Illinois, Urbana, Illinois 61801 